\title{
Cost-effectiveness analysis of 4 GLP-1RAs in the treatment of obesity in a US setting
}

\author{
Ying $\mathrm{Hu}^{1 \#}{ }^{11}$, Shui-Lian Zheng ${ }^{1 \#}$, Xiao-Lan Ye ${ }^{1}$, Jia-Na Shi ${ }^{1}$, Xiao-Wei Zheng ${ }^{1}$, Han-Sheng Pan ${ }^{1}$, \\ Yi-Wen Zhang $^{1,2}$, Xiu-Li Yang ${ }^{1 *}$, Ping Huang ${ }^{1,2 *}$ \\ ${ }^{1}$ Clinical Pharmacy Center, Department of Pharmacy, Zhejiang Provincial People's Hospital, Affiliated People's Hospital, Hangzhou Medical \\ College, Hangzhou, China; ${ }^{2}$ Key Laboratory of Endocrine Gland Diseases of Zhejiang Province, Hangzhou, China \\ Contributions: (I) Conception and design: Y Hu; (II) Administrative support: XL Yang, P Huang; (III) Provision of study materials or patients: XL \\ Ye, JN Shi; (IV) Collection and assembly of data: XW Zheng, HS Pan; (V) Data analysis and interpretation: SL Zheng, YW Zhang; (VI) Manuscript \\ writing: All authors; (VII) Final approval of manuscript: All authors. \\ \#These authors contributed equally to this work and should be regard as co-first authors. \\ *These authors contributed equally to this work. \\ Correspondence to: Xiu-Li Yang. Clinical Pharmacy Center, Department of Pharmacy, Zhejiang Provincial People's Hospital, Affiliated People's \\ Hospital of Hangzhou Medical College, Hangzhou 310014, China. Email: xiuli8245@163.com; Ping Huang. Clinical Pharmacy Center, Department \\ of Pharmacy, Zhejiang Provincial People's Hospital, Affiliated People's Hospital of Hangzhou Medical College, Hangzhou 310014 , China. \\ Email: huangping@hmc.edu.cn.
}

Background: The number of obese people continues to increase worldwide, and obesity-related complications add to every country's health burden. Consequently, new weight-loss medications, such as glucagon-like peptide-1 receptor agonists (GLP-1RAs), are attracting increasing attention. This study sought to assess the cost effectiveness for weight loss of 4 GLP-1RAs in adult patients with obesity in the United States.

Methods: Four GLP-1RA groups that received Liraglutide (1.8 mg QD), Semaglutide (1.0 mg QW), Dulaglutide (1.5 mg QW), or Exenatide (10 $\mathrm{g}$ BID), and one no-treatment group were compared using a decision-tree model. All the estimated parameters were derived from published articles. Quality-adjusted life years (QALYs), costs, and incremental cost-effectiveness ratios (ICERs) were adopted as the study endpoints. We analyzed the results with the willingness-to-pay (WTP) threshold, and conducted deterministic and probabilistic sensitivity analyses.

Results: The GLP-1RAs produced effective weight-loss results; however, not all the GLP-1RAs were cost effective compared to no treatment based on a WTP threshold of \$195000/QALY. Among the 4 GLP-1RAs, Semaglutide provided a cost-effective strategy with an ICER of \$135467/QALY. The sensitivity analyses showed that these results are reliable.

Conclusions: Among the 4 GLP-1RAs, Semaglutide was the most cost-effective obesity medication.

Keywords: Glucagon-like peptide-1 receptor agonists (GLP-1RAs); cost-effectiveness analysis; obesity; weight loss

Submitted Dec 21, 2021. Accepted for publication Jan 30, 2022.

doi: $10.21037 / \mathrm{atm}-22-200$

View this article at: https://dx.doi.org/10.21037/atm-22-200

^ ORCID: 0000-0002-6218-9051. 


\section{Introduction}

An estimated 650 million adults are overweight or obese, and the number of obese people continues to increase worldwide (1). In the United States (US), overweight or obesity rates have increased threefold in the last 40 years (2). As the burden of obesity increases, the incidence of obesityrelated health problems also increases. Obesity is a risk factor for chronic diseases, including hypertension, diabetes, and cardiovascular diseases, and is also an independent risk factor for mortality (3). Globally, it is estimated that obesityrelated complications will cost US\$1.2 trillion by 2025 , of which nearly half of this sum will be spent in the US. A $5 \%$ weight loss in obese patients can improve their health and reduce the incidence of obesity-related complications (4). Thus, effective treatments and interventions are essential for obese people. From the perspective of global public health, obesity is now classified as a disease that needs to be managed with high priority and is attracting attention around the world.

The current preferred treatment for obesity is lifestyle interventions, such as controlling diet and strengthening exercises. However, these measures further trigger the body's adaptive physiological responses, leading to increased appetite and a lower resting metabolic rate (5). Thus, it is difficult for obese people to achieve weight loss only through lifestyle interventions. Bariatric surgery is an effective treatment for obesity, but it is not the first choice because of its invasive nature. Conversely, pharmacotherapy is a non-invasive approach that can help people with obesity to adhere to weight-loss strategies, improve their quality of life, and reduce the risk of comorbidities (6). Glucagonlike peptide-1 receptor agonists (GLP-1RAs) are a class of therapeutic agents that provide significant improvements in Hemoglobin A1c (HbA1c), and also promote weight loss and carry a low risk of hypoglycemia (7-9). They delay gastric emptying, thereby increasing the duration of satiety, causing obese people to reduce their food intake and ultimately achieve weight loss. Liraglutide was approved by the European Medicines Agency for weight management pharmacotherapy in 2015, and in June 2021, the Food and Drug Administration (FDA) approved Semaglutide as a supplement to diet and exercise for the treatment of obesity (10). Dulaglutide and Exenatide are not approved by the FDA for the treatment of overweight individuals. However, the efficacy of both medications in terms of weight loss has been shown, and they are increasingly being used clinically (11-14).
A number of studies have evaluated the cost effectiveness of GLP-1RAs in the treatment of diabetes, and the efficiency parameter is mostly HbA1c, but only a few studies have evaluated their weight-loss efficacy (15). Our study focused on the cost effectiveness of GLP-1RAs in weight-loss, and used quality-adjusted life years transferred from the change of body mass index (BMI) as the efficiency parameter. It is difficult to conduct large-scale clinical trials to compare the effectiveness of various GLP-1RA treatments for obesity, as such trials need to meet many requirements, including having a huge sample size, and have high costs. This study sought to compare and analyze the cost-effectiveness of 4 GLP-1RAs in weight-loss efficacy to provide a reference for obesity control and medical cost savings. We present the following article in accordance with the CHEERS reporting checklist (available at https://atm. amegroups.com/article/view/10.21037/atm-22-200/rc).

\section{Methods}

\section{Model overview}

We developed a decision-tree model, using TreeAge Pro 2011 (TreeAge) to assess the cost effectiveness of the following 5 strategies: Liraglutide (1.8 $\mathrm{mg}$ QD), Semaglutide (1.0 mg QW), Dulaglutide (1.5 mg QW), Exenatide $(10 \mu \mathrm{g}$ BID), and no treatment. All the medications were administered by subcutaneous injections. TreeAge Pro is a software platform that is used to build and analyze state-transition models, which are useful for assessing the effect of treatments, including their cost effectiveness (16). The model was extended to half a year to estimate weight-loss efficiency. All the clinical data used in the analysis were based on 4 prospective clinical trials. In this model, all the patients kept active with the treatments until the study ended. Because this was a mathematical analysis and did not include any individual-level patient data, the Zhejiang Provincial People's Hospital Institutional Ethics committee waived the ethical approval and informed consent in this context. The study was conducted in accordance with the Declaration of Helsinki (as revised in 2013).

\section{Strategies for weight management}

According to published literature, the weight of untreated obese patients will continue to increase slightly over time (17). For patients treated with GLP-1RAs, the 
Table 1 Characteristics of populations used in the clinical trials.

\begin{tabular}{|c|c|c|c|c|c|c|c|c|c|}
\hline Trials & \multicolumn{2}{|c|}{ Zinman et al. } & \multicolumn{2}{|c|}{ Russell-Jones et al. } & \multicolumn{2}{|c|}{ Apovian et al. } & \multicolumn{2}{|c|}{ Wysham et al. } & $P$ value \\
\hline Sample Size & 150 & 151 & 230 & 114 & 96 & 98 & 163 & 83 & 0.210 \\
\hline Age (years) & $57.5(8.9)$ & $56.6(10.1)$ & $57.6(9.5)$ & $57.5(9.6)$ & $54.5(10.0)$ & $55.1(9.0)$ & $56(10.0)$ & $55(10.0)$ & 0.045 \\
\hline Male (\%) & $59 \%$ & $58 \%$ & $57 \%$ & $49 \%$ & $37 \%$ & $38 \%$ & $58 \%$ & $59 \%$ & 0.625 \\
\hline $\begin{array}{l}\text { Baseline } \\
\text { Weight (kg) }\end{array}$ & $89.6(19.5)$ & $93.8(22.3)$ & 85.5 (19.4) & $85.7(16.7)$ & 94.9 (16.5) & $96.2(15.6)$ & $96(20.0)$ & $94(19.0)$ & 0.160 \\
\hline BMI $\left(\mathrm{kg} / \mathrm{m}^{2}\right)$ & $31.1(6.2)$ & $32.7(6.9)$ & $30.4(5.3)$ & $31.3(5.0)$ & $33.6(3.7)$ & $33.9(4.3)$ & $33(5.0)$ & $33(6.0)$ & 0.094 \\
\hline
\end{tabular}

Values are mean (SD). ${ }^{*} \mathrm{P}>0.01$ indicates no significant difference between the four treatments. BMI, body mass index.

Table 2 Treatment effects and costs applied in the analyses.

\begin{tabular}{|c|c|c|c|}
\hline Treatment & Total $\triangle \mathrm{BMI}$ & $\begin{array}{c}\text { Monthly medication } \\
\text { costs, } \$\end{array}$ & $\begin{array}{c}\text { Daily needle } \\
\text { costs, } \$\end{array}$ \\
\hline No treatment & 0.0762 & 0 & 0 \\
\hline Semaglutide & -1.40 & 827.7 & 1.689 \\
\hline Liraglutide & -0.49 & 921.9 & 23.646 \\
\hline Exenatide & -0.79 & 729.6 & 11.823 \\
\hline Dulaglutide & -0.882 & 813.6 & 1.689 \\
\hline
\end{tabular}

$\mathrm{BMI}$, body mass index.

efficiency data were obtained from randomized, placebocontrolled clinical trials (18-21). There was no statistical difference in the basic population data of the 4 clinical trials (see Table 1). All the clinical trials included half-year weightloss data. The average baseline weight and body mass index (BMI) values in the experimental cohort were used to convert weight changes into BMI change rates (see Table 2).

\section{Costs and QOL adjustments}

The data of weight change were transferred to qualityadjusted life years (QALYs). According to previous research, a unit BMI loss leads to a gain of 0.0056 QALYs $(16,21)$. It was assumed from the perspective of the healthcare system that the cost of the no-treatment group was zero, and the cost of the GLP-1RAs groups were based on published literature (see Table 2) (22). For all the medication groups, the cost of 2 doctor visits (\$178) before the patients started taking the weight-loss medications was added (23). The cost of the injection needle used was also based on the previous literature (22). Costs for obesity-related comorbidities and adverse events caused by medications were not included. All the costs in this study were adjusted to 2019 US dollars. Costs and utilities were discounted at a rate of $3 \%$.

\section{Statistical analysis \& outcomes}

QALYs, costs, and incremental cost-effectiveness ratios (ICERs) were adopted as the study endpoints. ICERs were calculated based on the ratio of cost and QALY differences between the strategy and the suboptimal option. The willingness-to-pay (WTP) threshold of \$195,000/QALY was used to determine the cost effectiveness of the drugs, which came from the threefold of gross domestic product per capita in the US in 2019.

\section{Sensitivity analyses}

A deterministic analysis and probabilistic sensitivity analysis (PSA) were performed to assess the effect of parameter uncertainty on the cost-effectiveness results. one parameter at a time was varied within the prescribed bounds to perform the deterministic one-way sensitivity analyses, and the variations in ICERs were recorded. The cost parameters were varied by $+/-3 \%$ of the base-case values, and the efficiency parameters were varied by a confidence interval of $95 \%$ based on the clinical trial data. To conduct the PSA, all the parameters of probability distributions were sampled simultaneously. In the PSA, the cost parameter used the Gamma distribution, and other parameters, such as efficacy, used the Beta distribution. The mean value in the distribution was the basic case value, and the standard deviation parameter was derived from the clinical trial results. 1,000 Monte Carlo samples were run for each 
Table 3 Base-case results

\begin{tabular}{lccc}
\hline Treatment & Total costs, $\$$ & $\Delta$ QALYs & ICER, \$/QALY \\
\hline No treatment & 0 & -0.0002 & - \\
Semaglutide & 5137.32 & 0.0083 & 135467 \\
Liraglutide & 5772.30 & 0.0032 & Dominated \\
Exenatide & 4674.57 & 0.0048 & 982032 \\
Dulaglutide & 5052.33 & 0.0053 & 733243 \\
\hline
\end{tabular}

QALY, quality-adjusted life year; ICER, incremental cost-effectiveness ratio.

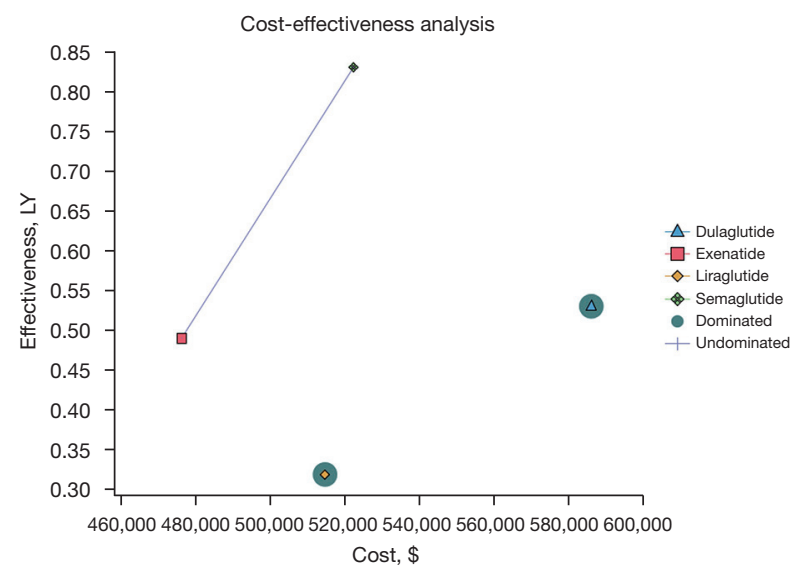

Figure 1 Base-case results of cost-effectiveness planes. The dashed lines indicate the efficiency frontiers.

group. The percentage of times at WTP for each GLP1RA was used to determine its cost effectiveness.

\section{Results}

\section{Base-case results}

Table 3 sets out the base-case analysis results. No treatment was regarded as the reference strategy because it had the lowest costs and the lowest effectiveness. In terms of cost effectiveness, Exenatide and Semaglutide was the relative cost-effective strategy on the efficiency frontier after half a year, dominating all other strategies. The ICER for Exenatide was \$982,032/QALY, the ICER for Semaglutide was $\$ 135,467 /$ QALY, and the ICER for Dulaglutide was $\$ 733,243 /$ QALY. Based on a willingness-to-pay (WTP) threshold of \$195,000/QALY, Exenatide, Dulaglutide and Semaglutide were not cost effective.

However, if Exenatide was the reference strategy (i.e., had the lowest cost), Semaglutide, with an ICER of $\$ 135,467 /$ QALY, was the most cost-effective strategy. That is to say, Semaglutide was the most effective treatment in terms of weight loss. The efficiency boundary plots the cost and effectiveness of each GLP-1RA (see Figure 1). The optimal strategy is located at the efficiency boundary (dashed line), and the suboptimal (inferior) strategy is located below the boundary. Dulaglutide was not a cost-effective strategy based on the results, as it had an ICER of \$733,243/QALY.

\section{One-way sensitivity results}

The results of the one-way sensitivity analyses are shown in Figure 2. Two GLP-1RAs (i.e., Semaglutide and Exenatide) were compared on the efficiency frontier. BMI loss parameter of Exenatide, the costs of Semaglutide and Exenatide had a greater effect on the results than other parameters, and only changing the rate of Exenatide BMI loss had a substantial effect on the resulting Semaglutide ICER. Exenatide became the most cost-effective strategy when the BMI loss for patients using Exenatide was over $0.97 \mathrm{~kg} / \mathrm{m}^{-2}$. The one-way sensitivity analysis results showed the base-case results were reliable, and Semaglutide remained the cost-effective GLP-1RA under varying conditions. The ICER of Semaglutide was below the WTP threshold in all the scenario-tests, unless Exenatied BMI loss changed.

\section{PSA results}

The PSAs of the 4 GLP-1RAs were performed using the model. The results included acceptability curves (see Figure 3) and scatter plots (see Figure 4). The analyses adopted the latest WTP threshold of $\$ 195,000$ per QALY gained proposed for the US. After half a year, Semaglutide was the most cost-effective choice in $75.3 \%$ of runs, and Exenatide in $24.7 \%$ of runs. Semaglutide and Exenatide were compared, and as Figure 5 shows, Semaglutide was more cost effective than Exenatide, with a probability of $76.9 \%$.

\section{Discussion}

Currently, very few weight-loss drugs have been approved for use by the FDA, other than Lorcaserin and Orlistat, which have relatively modest clinical efficacy and have not been widely used in clinical practice or well known to the public (24). Additionally, phentermine is not the best 


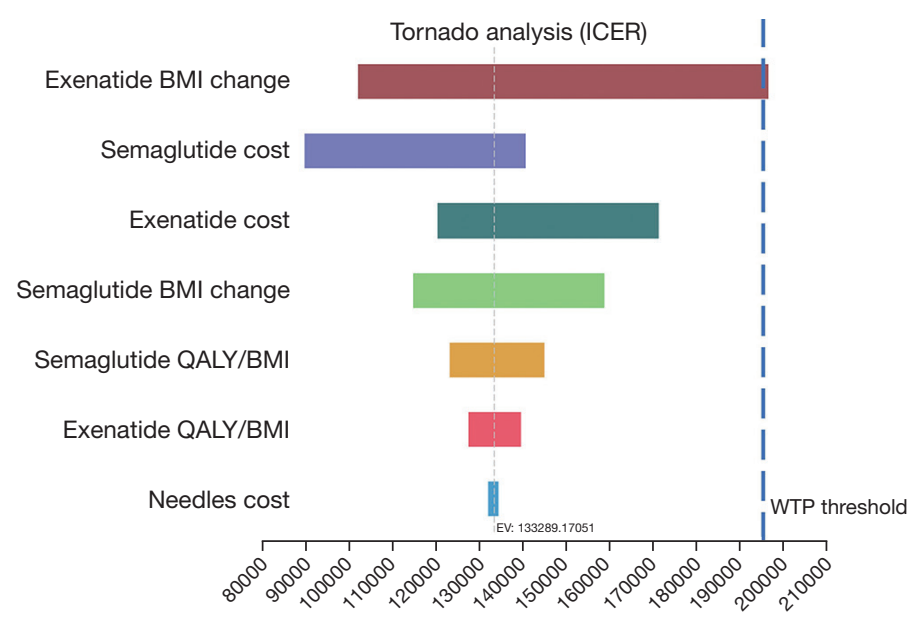

Figure 2 Tornado diagrams comparing Exenatide vs. Semaglutide in a one-way sensitivity analysis ranged in Semaglutide ICERs. ICER, incremental cost-effectiveness ratio. WTP, willingness-to-pay.

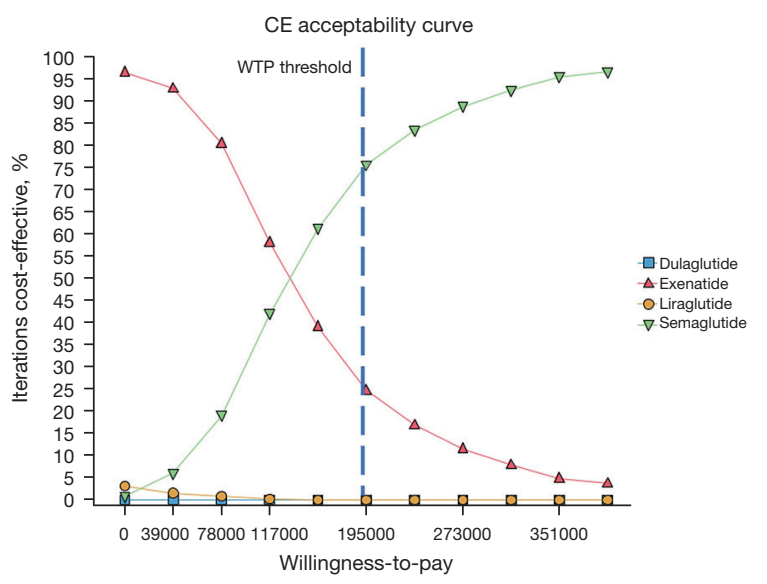

Figure 3 PSA results depicted as acceptability curves. The WTP threshold is indicated by the dashed line in the base case. WTP, willingness-to-pay. PSA, probabilistic sensitivity analysis.

choice for cardiovascular disease patients because of its adverse effects even if it has a better curative effect (25). Thus, GLP-1RAs bring hope to obese people. GLP-1RAs were originally developed to control blood glucose, but almost all clinical trials containing GLP-1RAs have found that they have a weight-loss effect, and some studies have focused on evaluating the weight-loss effect of GLP-1RAs in various types of obese people (e.g., GLP-1RAs have produced positive results in individuals with polycystic ovary syndrome) $(26,27)$. Previous studies on the cost effectiveness of GLP-1Ras have focused on glycemic control in diabetic patients (15), but this study is the first to

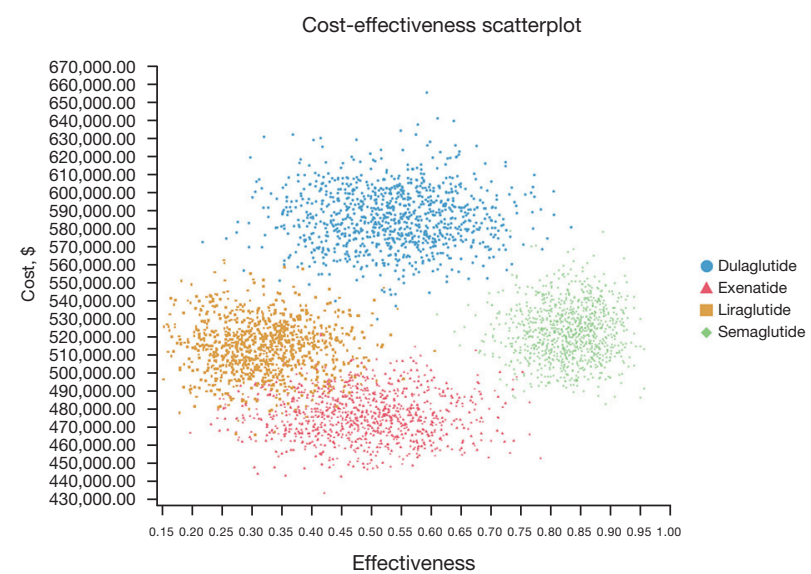

Figure 4 Acceptability curves of PSAs for cost-effectiveness planes. PSA, probabilistic sensitivity analysis.

conduct an economic analysis on the weight-loss effects of multiple GLP-1RAs. Additionally, GLP-1RAs are suitable for patients with cardiovascular diseases because of their cardiovascular protective effects.

As GLP-1RAs are novel medications with high price tags, our results suggest that GLP-1RAS are not economical alternative measures for weight loss. However, given that there are few weight-loss medications that can be applied clinically at present, and that most such medications have unsatisfactory efficacy or more serious side effects, GLP-1RAs represent relatively safe and more effective medications for many obese people. Among the GLP-1RAs, 


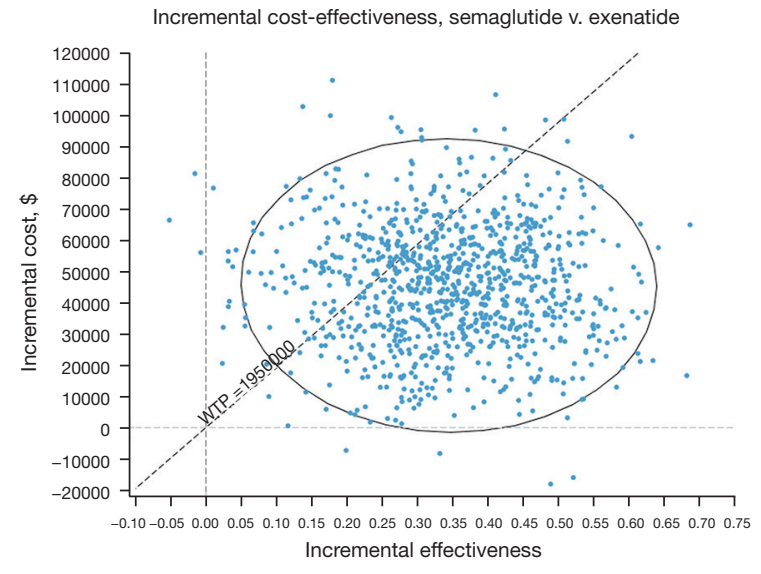

Figure 5 Scatter plot of PSA results for Semaglutide and Exenatide. PSA, probabilistic sensitivity analysis.

several weight-loss programs have shown the effect of Exenatide on weight loss in individuals with hypothalamic obesity and clozapine-associated obesity $(28,29)$. Semaglutide, the latest GLP-1RA, has a relatively higher price and a significantly higher weight-loss effect than Exenatide. Our modelling results found that Semaglutide $(1 \mathrm{mg})$ was associated with the lowest costs per patient in achieving treatment targets of weight loss among the 4 GLP-1RAs. Thus, this intervention might be the most costeffective GLP-1RA in the US in terms of enabling patients to achieve obesity control goals. Additionally, Semaglutide is currently available in oral formulation, and the effect and price are similar to the injection formulation (30). As the most efficacy and economical medication in weightloss among the four GLP-1RAs, it is likely that more obese people will choose Semaglutide.

Medication-associated adverse events were not examined in this study, which is a limitation of this study. The QALY was only calculated based on weight changes. This was because no apparent differences in the incidence of major adverse reactions, such as hypoglycemia and gastrointestinal reactions, have been observed for these 4 GLP-1RAs. The effect of cost changes in the sensitivity analysis also confirmed that a certain degree of cost changes would not have a significant effect on the results. Additionally, as the clinical trials used in this study only included half-year data, the cost-effectiveness analysis focused on the shortterm effects of weight loss. This is because a majority of obese people stop using medications after a certain degree of weight loss. The shedding rate of the obese population is high. Moreover, many studies have found that a plateau or weight re-gain occurs after about 40 weeks $(31,32)$. Therefore, this study only examined the short-term cost effectiveness of the GLP-1RAs. Also, the issue of intermittent medication taking was not considered in this study.

In summary, we found that Semaglutide is the most costeffective medication for weight loss among the 4 GLP1RAs examined. Recently, phase II clinical data have been published on a peptide with a potent balanced co-agonism for both the GLP-1 and glucose-dependent insulinotropic polypeptide that showed that it has a better weight-loss effect and may also be sold at a higher price (33). This highlights the need for further research into other new medications from additional clinical trials to fully examine the changes of weight, weight-related comorbidities, and associated adverse events.

\section{Acknowledgments}

Funding: This study was supported by "10000 Talents Plan” of Zhejiang Province to Ping Huang(grant No. 2020R52029), the Zhejiang Provincial Natural Science Foundation of China (grant No. LYY21H310011), Zhejiang Provincial Program for the Cultivation of New Heath Talents to Yiwen Zhang, Natural Science Foundation of Zhejiang Province (grant No. LYY19H310009), Zhejiang Pharmaceutical Association Foundation (grant No. 2020ZYY22), Foundation of Education of Zhejiang Province (grant No. Y202146108), Zhejiang Pharmaceutical Association Foundation (grant No. 2018ZYY30), Medical and Health Research Program of Zhejiang Province (grant No. 2022KY063), Medical and Health Research Program of Zhejiang Province (grant No. 2019KY273), National Natural Science Foundation of China (grant No. 82003189), Natural Science Foundation of Zhejiang Province (grant No. LQ17H310002), and Research Program from Education of Zhejiang Province (grant No. Y202146108).

\section{Footnote}

Reporting Checklist: The authors have completed the CHEERS reporting checklist. Available at https://atm. amegroups.com/article/view/10.21037/atm-22-200/rc

Data Sharing Statement: Available at https://atm.amegroups. 
com/article/view/10.21037/atm-22-200/dss

Conflicts of Interest: All authors have completed the ICMJE uniform disclosure form (available at https://atm. amegroups.com/article/view/10.21037/atm-22-200/coif). The authors have no conflict of interests to declare.

Ethical Statement: The authors are accountable for all aspects of the work in ensuring that questions related to the accuracy or integrity of any part of the work are appropriately investigated and resolved. The study was conducted in accordance with the Declaration of Helsinki (as revised in 2013). Because this was a mathematical analysis and did not include any individual-level patient data, the Zhejiang Provincial People's Hospital Institutional Ethics committee waived the ethical approval and informed consent in this context.

Open Access Statement: This is an Open Access article distributed in accordance with the Creative Commons Attribution-NonCommercial-NoDerivs 4.0 International License (CC BY-NC-ND 4.0), which permits the noncommercial replication and distribution of the article with the strict proviso that no changes or edits are made and the original work is properly cited (including links to both the formal publication through the relevant DOI and the license). See: https://creativecommons.org/licenses/by-nc-nd/4.0/.

\section{References}

1. Seidell JC, Halberstadt J. The global burden of obesity and the challenges of prevention. Ann Nutr Metab 2015;66 Suppl 2:7-12.

2. Xu H, Cupples LA, Stokes A, et al. Association of Obesity With Mortality Over 24 Years of Weight History: Findings From the Framingham Heart Study. JAMA Netw Open 2018;1:e184587.

3. An S, Park HY, Oh SH, et al. Cost-effectiveness of Bariatric Surgery for People with Morbid Obesity in South Korea. Obes Surg 2020;30:256-66.

4. Visscher TL, Seidell JC. The public health impact of obesity. Annu Rev Public Health 2001;22:355-75.

5. Saunders KH, Umashanker D, Igel LI, et al. Obesity Pharmacotherapy. Med Clin North Am 2018;102:135-48.

6. Yumuk V, Tsigos C, Fried M, et al. European Guidelines for Obesity Management in Adults. Obes Facts 2015;8:402-24.

7. Monami M, Dicembrini I, Marchionni N, et al. Effects of glucagon-like peptide-1 receptor agonists on body weight: a meta-analysis. Exp Diabetes Res 2012;2012:672658.

8. Vilsbøll T, Christensen M, Junker AE, et al. Effects of glucagon-like peptide-1 receptor agonists on weight loss: systematic review and meta-analyses of randomised controlled trials. BMJ 2012;344:d7771.

9. Sun F, Chai S, Li L, et al. Effects of glucagon-like peptide-1 receptor agonists on weight loss in patients with type 2 diabetes: a systematic review and network metaanalysis. J Diabetes Res 2015;2015:157201.

10. European Medicines Agency. Saxenda Sum- mary of Product Characteristics. Available online: https://www. ema.europa.eu/en/documents/product-information/ saxendaeparproduct-information_en.pdf Accessed 2021 May 18.

11. Taylor K, Gurney K, Han J, et al. Exenatide once weekly treatment maintained improvements in glycemic control and weight loss over 2 years. BMC Endocr Disord 2011;11:9.

12. Tham LS, Pantalone KM, Dungan K, et al. A modelbased simulation of glycaemic control and body weight when switching from semaglutide to $3.0-$ and $4.5-\mathrm{mg}$ doses of once-weekly dulaglutide. Diabetes Obes Metab 2022;24:302-11.

13. Bonora E, Frias JP, Tinahones FJ, et al. Effect of dulaglutide 3.0 and $4.5 \mathrm{mg}$ on weight in patients with type 2 diabetes: Exploratory analyses of AWARD-11. Diabetes Obes Metab 2021;23:2242-50.

14. Lazzaroni E, Ben Nasr M, Loretelli C, et al. Anti-diabetic drugs and weight loss in patients with type 2 diabetes. Pharmacol Res 2021;171:105782.

15. Htike ZZ, Zaccardi F, Papamargaritis D, et al. Efficacy and safety of glucagon-like peptide-1 receptor agonists in type 2 diabetes: A systematic review and mixed-treatment comparison analysis. Diabetes Obes Metab 2017;19:524-36.

16. Klebanoff MJ, Chhatwal J, Nudel JD, et al. Costeffectiveness of Bariatric Surgery in Adolescents With Obesity. JAMA Surg 2017;152:136-41.

17. Malhotra R, Ostbye T, Riley CM, et al. Young adult weight trajectories through midlife by body mass category. Obesity (Silver Spring) 2013;21:1923-34.

18. Zinman B, Bhosekar V, Busch R, et al. Semaglutide once weekly as add-on to SGLT-2 inhibitor therapy in type 2 diabetes (SUSTAIN 9): a randomised, placebo-controlled trial. Lancet Diabetes Endocrinol 2019;7:356-67.

19. Russell-Jones D, Vaag A, Schmitz O, et al. Liraglutide vs insulin glargine and placebo in combination with metformin and sulfonylurea therapy in type 2 diabetes 
mellitus (LEAD-5 met+SU): a randomised controlled trial. Diabetologia 2009;52:2046-55.

20. Apovian CM, Bergenstal RM, Cuddihy RM, et al. Effects of exenatide combined with lifestyle modification in patients with type 2 diabetes. Am J Med 2010;123:468.e9-17.

21. Wysham C, Blevins T, Arakaki R, et al. Efficacy and safety of dulaglutide added onto pioglitazone and metformin versus exenatide in type 2 diabetes in a randomized controlled trial (AWARD-1). Diabetes Care 2014;37:2159-67.

22. Hansen BB, Nuhoho S, Ali SN, et al. Oral semaglutide versus injectable glucagon-like peptide-1 receptor agonists: a cost of control analysis. J Med Econ 2020;23:650-8.

23. Finkelstein EA, Kruger E, Karnawat S. Cost-Effectiveness Analysis of Qsymia for Weight Loss. Pharmacoeconomics 2015;33:699-706.

24. Lee M, Lauren BN, Zhan T, et al. The cost-effectiveness of pharmacotherapy and lifestyle intervention in the treatment of obesity. Obes Sci Pract 2019;6:162-70.

25. Lewis KH, Fischer H, Ard J, et al. Safety and effectiveness of longer-term phentermine use: clinical outcomes from an electronic health record cohort. Obesity (Silver Spring) 2019;27:591-602.

26. Elkind-Hirsch KE, Chappell N, Seidemann E, et al. Exenatide, Dapagliflozin, or Phentermine/Topiramate Differentially Affect Metabolic Profiles in Polycystic Ovary Syndrome. J Clin Endocrinol Metab 2021;106:3019-33.

27. Nowrouzi-Sohrabi P, Rezaei S, Jalali M, et al. The effects of glucagon-like peptide-1 receptor agonists on glycemic control and anthropometric profiles among diabetic patients with non-alcoholic fatty liver disease: A systematic review and meta-analysis of randomized controlled trials. Eur J Pharmacol 2021;893:173823.

28. Roth CL, Perez FA, Whitlock KB, et al. A phase 3 randomized clinical trial using a once-weekly glucagonlike peptide-1 receptor agonist in adolescents and young adults with hypothalamic obesity. Diabetes Obes Metab 2021;23:363-73.

29. Siskind DJ, Russell AW, Gamble C, et al. Treatment of clozapine-associated obesity and diabetes with exenatide in adults with schizophrenia: A randomized controlled trial (CODEX). Diabetes Obes Metab 2018;20:1050-5.

30. Pratley R, Amod A, Hoff ST, et al. Oral semaglutide versus subcutaneous liraglutide and placebo in type 2 diabetes (PIONEER 4): a randomised, double-blind, phase 3a trial. Lancet 2019;394:39-50.

31. Weinstock RS, Guerci B, Umpierrez G, et al. Safety and efficacy of once-weekly dulaglutide versus sitagliptin after 2 years in metformin-treated patients with type 2 diabetes (AWARD-5): a randomized, phase III study. Diabetes Obes Metab 2015;17:849-58.

32. Henry RR, Klein EJ, Han J, et al. Efficacy and Tolerability of Exenatide Once Weekly Over 6 Years in Patients with Type 2 Diabetes: An Uncontrolled Open-Label Extension of the DURATION-1 Study. Diabetes Technol Ther 2016;18:677-86.

33. Bhagavathula AS, Vidyasagar K, Tesfaye W. Efficacy and Safety of Tirzepatide in Patients with Type 2 Diabetes Mellitus: A Systematic Review and Meta-Analysis of Randomized Phase II/III Trials. Pharmaceuticals (Basel) 2021;14:991.
Cite this article as: $\mathrm{Hu} \mathrm{Y,} \mathrm{Zheng} \mathrm{SL,} \mathrm{Ye} \mathrm{XL,} \mathrm{Shi} \mathrm{JN,}$ Zheng XW, Pan HS, Zhang YW, Yang XL, Huang P. Costeffectiveness analysis of 4 GLP-1RAs in the treatment of obesity in a US setting. Ann Transl Med 2022;10(3):152. doi: 10.21037/atm-22-200 